\title{
THE BLOCKCHAIN TECHNOLOGY AND CLOUD COMPUTING IMPACT FOR CRYPTOECONOMY CONTEXT
}

\author{
Dan-Cristian CEARNĂU \\ Bucharest University of Economic Studies, Romania \\ dan.cearnau@udevoffice.ro
}

\begin{abstract}
In the human evolution, the man evolved alongside society in a series of revolutions that led to great improvements on all domains: cultural, social, industrial, etc. Today we are living what futurologist as Alvin Toffler in "Future Shock" and more recently Michael Dertouzos in "What Will Be: How the New World of Information Will Change Our Lives" anticipated more than 20, respectively 50 years ago. They imagined a world in which the information technology is based on learning.

Today, technological singularity is no longer just a concept, it's a reality. The technical and scientific progress that has materialized in real revolutions has recorded not only a one-way amplification movement, but perhaps even an acceleration. Today, on the 30th anniversary of the World Wide Web, Information and Communication Technology (ICT) has become not only a neo-factor of production for most businesses but the indispensable factor of any activity. This is the context in which a new technological revolution - the Blockchain revolution - takes place in the face of an "apparent financial crisis" in 2008-2009 and a lower level of confidence. The advantages of this technology, coupled with another relatively modern development (as an implementation, not as a concept) of ICT, namely cloud computing, are now leading to increasingly spectacular solutions in the digital economy, electronic money, e-Government, or electronic citizen (e-citizen). In this paper we will be able to deal with the impact of the two technologies in the context of the digital economy, materialized in at least two paradigms: Blockchain as a Service (BaaS) and Blockchain as a Cloud (BaaC).
\end{abstract}

Keywords: blockchain, cryptocurrency, cloud computing, blockcloud, Blockchain-as-aService (BaaS), thin cloud, ICT.

JEL classification: $\mathrm{O} 3$

DOI: $10.12948 / \mathrm{ie} 2019.02 .07$

\section{Cloud Computing}

According to NIST (National Institute of Standards and Technologies), cloud computing is an advanced calculation model for convenient, on-demand and remote access over the Internet to a configurable set of shared resources (networks, servers, external memory, services). These resources can be delivered quickly with minimal administrative effort and minimum interaction with the provider. There are three basic cloud models [1]:

IaaS (Infrastructure as a Service) is the lowest level of abstraction. The user is provided with complete computing infrastructure (processors, memories, storage space, bandwidth, servers) in the form of a virtual machine. These virtual machines are created on demand and destroyed when they are no longer needed by designated software agents and hypervisors. Customers can allocate their own resources using Ucloud Middleware Programs or cloud management systems (CMS). Examples are Amazon Web Services EC2, Google Compute Engine, VMWarevCloud. 
PaaS (Platform as a Service) is an intermediate level. Platforms are built on IaaS foundations and are offered to customers along with development tools and environments. Examples are Microsoft Azure, Google App Engine, and Apache.

\section{Blockchain}

A blockchain is basically a distributed database of records or a publication of all transactions (digital events) that have been executed and divided between the participating entities. Each transaction in the public register is verified by the consensus of most participants in the system. Once entered, the information cannot be deleted. The block contains a secure and verifiable record of each transaction ever made (Figure 2). To use a basic analog, it's easy to steal a cookie from a cookie jar kept in a secured place rather than stealing the cookie from a cookie jar kept in a market, of thousands of people. There are three different types of blocks:

- public: such as Bitcoin, are large distributed networks that run through a native symbol. They are open for anyone to participate at any level and have an open source code that the community maintains.

- permission: such as Ripple, control roles that individuals can play on the network. There are still large and distributed systems that use a native token. Their basic code may or may not be open source.

- private: tend to be smaller and not use a token.

Their belonging is closely controlled. These types of blocks are favored by consortiums that trust members and trade confidential information. All three types of blocks use cryptography to allow each participant in any given network to manage the registry in a safe way without the need for a central authority to impose rules. The removal of the central authority from the database structure is one of the most important and powerful aspects of the blocks. [2]

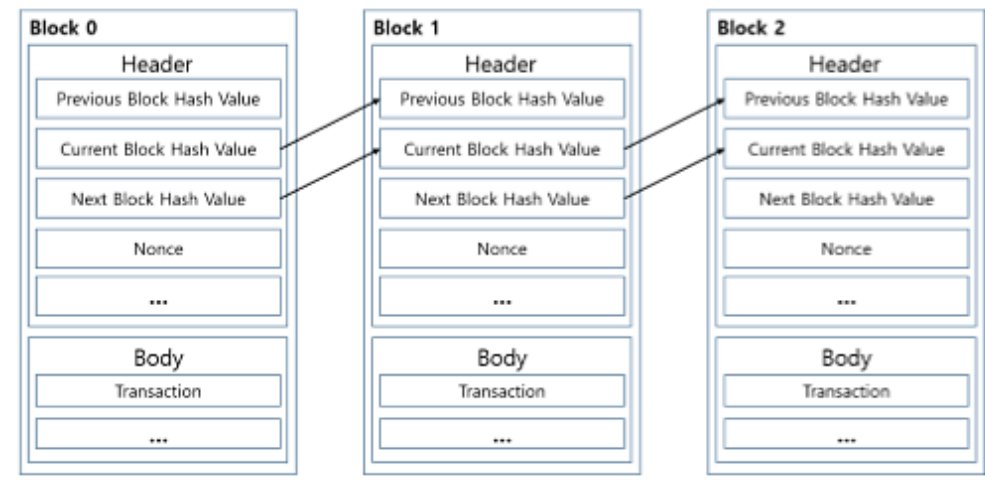

Figure 7 - Blockchain, connection structure (adaptation from [8])

As a peer-to-peer network combined with a time-sharing server, the blockchain databases can be managed autonomously to exchange information between disparate parts. There is no need for an administrator, blockchain users being the administrator. In addition, blockchain networks can be used for "smart contracts" or for scripts that run automatically when certain conditions are met. For example, Ethereum users must meet pre-established conditions that prove someone owns a certain token and have the authority to send the money that they claim to have. Multiple blockchain users can create contracts that require more than one set of entries to trigger a transaction. For example, real estate transactions require signatures between buyers, sellers and their financial institutions.

\section{Bitcoin}


Bitcoin is the most popular example that is intrinsically linked to blockchain technology, being virtually the first cryptocurrency from the digital financial market. It is also the most controversial, as it helps create a multibillion-dollar global anonymous transaction market without any government control. Therefore, it has to deal with a series of regulatory issues involving national governments and financial institutions. As I said, bitcoin is a digital coin launched in 2009, amid a deep financial crisis, by a mysterious person known only by the nickname Satoshi Nakamoto. Unlike the traditional currencies issued by central banks, the bitcoin has no central monetary authority. No one controls it. Bitcoins are not printed as dollars or euros; they are "mined" by people and more and more by businesses that run computers around the world using software that solves mathematical puzzles. Instead of relying on a central monetary authority to monitor, verify and approve transactions and manage money, bitcoin is activated by a computerized computer network made up of its users' machines, similar to the underlying BitTorrent and Skype networks. Bitcoin has several advantages over other current transaction systems. [3]

- Cost: bitcoin removes the need for a middle-man;

- Efficiency: transaction information is recorded once and is available to all parties through the distributed network;

- Secure: a transaction cannot be changed; this can only be reversed with another transaction, in which case both transactions are visible.

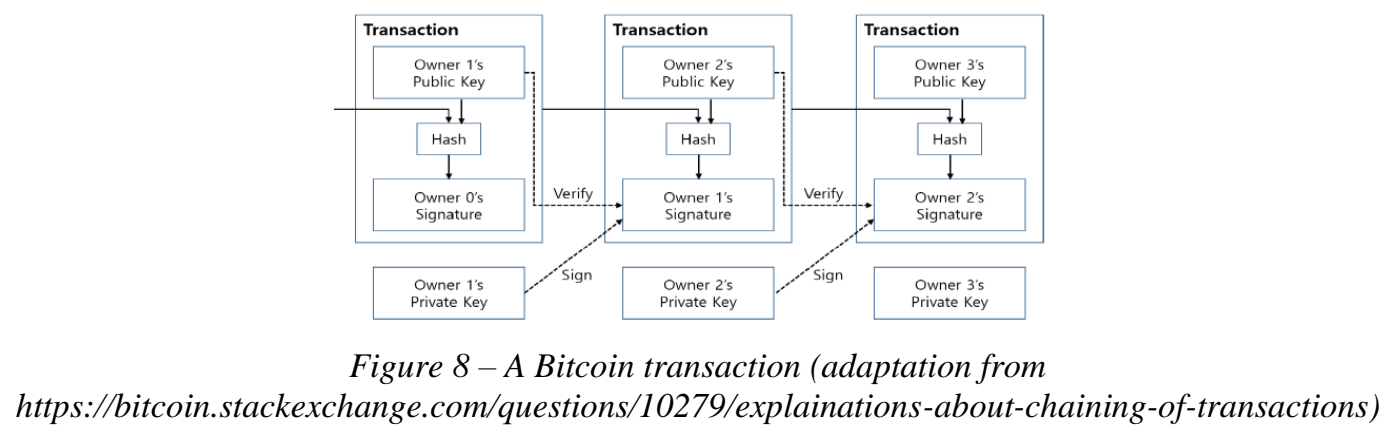

Bitcoin is actually built on the blockchain foundation, which serves as a common bitcoin registry. Think of the blockchain as an operating system, such as Microsoft Windows or MacOS, and bitcoin as just one of the many applications that can be run on that operating system. Blockchain provides the means to record bitcoin transactions - the shared registry - but this common registry can be used to record any transaction and track the movement of any items, whether tangible, intangible or digital.

For example, the blockchain allows securities to be capitalized in minutes rather than days. It can also be used to help companies manage the flow of goods and related payments, or allow manufacturers to divide production houses with original equipment manufacturers and regulators to reduce product withdrawals.

Bitcoin and blockhain are not the same. Blockchain provides the means of recording and storing bitcoin transactions, but the blockchain has many uses beyond bitcoin. Bitcoin is just the first case of use for the blockchain. The electronic coin used in bitcoin consists of an electronic signature chain, Figure 3)

The cryptocurrencies of an owner are transferred to the next chain with the hash value of the previous transaction, and the electronic signature is passed to the public key of the next owner. The addressee can check the signature to confirm the property chain. In this process, there is a problem: the recipient is not able to ensure that one of the owners has not used the currency (s) 
several times. A reliable central authority is introduced to verify all dual-use transactions to resolve this issue.

\section{Blockchain vs. Cloud}

Cloud technologies are the blockchain's predecessors, and developers who offer innovations in this area should also take account of blockchain opportunities. Blockchain private networks could be run in cloud security environments, which could therefore play a key role in blockchain implementations. At the same time, the cloud and the blockchain have so many common issues that the deployment of cloud blockchain pilot projects would ensure that many of their advantages are put into motion in one shot [4].

Cloud and blockchain have security protection systems, allowing total data encryption. The ability of cloud deployment models to explicitly address private, community, and public scenarios fits perfectly with the nature of the blocks, driven by dedicated chain design members. Both are resistant to cybercrime. Blockchain qualifies for this because of its peer-topeer model, while cloud is shielded from efficiently evolving and actively contributing to the creation of cyber-free areas. This includes 24-hour monitoring and proactive identification of suspicious activities and real-time response to such threats. Both cloud and blockchain significantly reduce costs. Blockchain, as well as cloud, avoids potential design inefficiencies from its processes.

Before companies such as Amazon could provide cloud services, the storage infrastructure was very decentralized. Companies hosted their own servers in their own offices. This has allowed greater control but also higher costs. Companies need internal expertise to configure and maintain costly servers and units. They also had to invest in backups outside the redundancy space. It is no surprise that most organizations have gone to the cloud. At about $\$ 25$ per terabyte a month, services such as the Amazon S3 offer incredible value and, by replicating data across multiple data centers, provide you with reliable time and redundancy. This convenience has blinded us from the disadvantages. When we use a cloud service, we put tremendous confidence in third parties. We trust these third parties to ensure our most sensitive and most private data. These data are usually unencrypted. Moreover, costs seem small, but that's because we do not have a good point of reference.

A blockchain allows the creation of a decentralized and distributed storage market. The most successful markets identify insufficient resources and meet the growing demand. Uber, for example, corresponded to underused cars, and consumers demanded better transport options. On a block storage market, hosts sell their storage capacity excessively, and tenants acquire this surplus capacity and upload files. The files are encrypted, fragmented and intelligently distributed in dozens of nodes. Using a jam allows a few things that were previously impossible: complete decentralization and true redundancy. Amazon S3 performs redundancy by distributing files to regional data centers. This makes each data center an important point of failure. In a decentralized block, where data is stored on dozens of individual nodes, intelligently disposed across the globe, it is extremely difficult to cause significant disturbances. Total security. No third party controls user data or has access to user files. Each node stores only encrypted user data fragments.

\section{Blockchain as a Service (BaaS)}

Blockchain is a transformation technology with the potential to expand digital transformation beyond the walls of a company and into the processes it shares with vendors, customers and partners. At its center, a block is a data structure used to create a digital transaction log, which, instead of resting at a single provider, is shared between a distributed network of computers. 
The result is a more open, transparent and verifiable system that will fundamentally change the way we think about exchanging values and assets, imposing contracts and exchanging data.

An increasing number of businesses invest in blockchain as a secure and transparent way of digitally tracking property ownership over assets beyond the confines of confidence. They are redesigning shared business processes, and create new cross-organizational collaboration patterns. Many of the cloud leaders have seen the potential benefits offered to customers by Blockchain as a service (BaaS) and have begun to provide a certain level of BaaS capabilities [5]:

- Microsoft (Azure) - In November 2016, Microsoft gave the first shot. They have announced a partnership with ConsenSys to provide Blockchain Ethereum as a Service (EBaaS) in the Azure environment. Delivering the service will allow "customers and partners to play, learn and succeed at a low cost in a dev / test / production ready production environment," says Marley Gray, Microsoft Cloud and Enterprise. As an open, flexible and scalable platform, Azure supports a growing number of distributed technologies that meet the specific business and technical requirements for security, performance and operational processes. Azure provides a fast, low-cost and low-risk platform for collaborating organizations experiencing new business processes - and is supported by a cloud platform with the industry's highest compliance portlet.

- IBM (BlueMix) - In February 2017, IBM announced that it would offer Blockchain as a service using Hyperledger. "With the help of the new IBM blockchain services available on Bluemix, developers can access fully integrated DevOps tools for building, deploying, running and monitoring Blockchain applications on IBM Cloud." Hyperledger is an open source collaboration effort designed to promote breakthrough technologies like blockchain. It is a global collaboration hosted by the Linux Foundation, including leaders in finance, banking, IoT, supply chain, manufacturing and technology. These 130+ members and 8 ongoing projects, including Hyperledger Fabric and Hyperledger Composer, work together to create an open and standardized framework for businesses and the code base.

- Amazon (AWS) - In May 2017, Amazon announced a collaboration with the Digital Currency Group, one of the largest investors in blockchain companies. The deal will provide Blockchain-as-a-Service to DCG portfolio members so they "can work in a secure environment with customers including financial institutions, insurance companies, and enterprise technology companies." AWS collaborates with financial institutions and blockchain technology suppliers to stimulate innovation and facilitate seamlessly experimentation. Scott Mullins, head of the financial affairs development department worldwide, said, "DCG is a recognized leader in distributed blockchain technology and we are delighted to work with them and with portfolio companies to provide laboratory environments for enterprise experimentation with blockchain technology on AWS ".

\section{Blockchain-as-a-Cloud}

Blockchain is a "thin cloud" compared to a traditional cloud computing infrastructure, and is therefore more suited to running new types of "thin" programs, specifically called "smart contracts," which are the logic of business executes on blockchain virtual machines (VM). Blockchain infrastructure does not replace cloud computing. It separates and democratizes certain parts of it. Moreover, the blockchain infrastructure resembles a cloud computing infrastructure layer. When running a cloud application (for example, Amazon Web Services or Microsoft Azure), it will be billed for a combination of time, storage, data transfer, and computing speed requirements. The innovation with the cost of the virtual machine (for example, on Ethereum) is that it pays to run business logic on the blockchain that actually runs 
on physical servers, but you do not have to worry about setting them up because they are managed by others users who are paid for running the infrastructure [6].

Therefore, Blockchain Cloud has a form of pricing model at micro-value, which is parallel to the traditional cloud computing stack, but with a new layer. But here is the challenge to run applications on this new infrastructure: something needs to be done. This work comes in the form of adhering to a new paradigm of decentralized applications following a new architecture created by Gavin Wood as "web3". Web3 is an architecture that runs specifically on blockchain. Using Ethereum as a primary example, a web3 architecture includes an advanced client browser, blockchain register as a shared resource, and a virtual computer network running smart business logic programs in a decentralized manner [7]. This new paradigm exemplifies the future direction of decentralized cryptographic computerization. It is a variant of the existing Web application architecture, which consists of running JavaScript inside the browsers and the server code running on the company's servers.

\section{Conclusions}

It seems that blockchain technology is like any other software technology, but will it be in the field of information technology departments? Yes, there will be some applications that will require the involvement of IT teams and a large suite of software to produce end-user, backend, intra- or inter-company applications. But a more disturbing aspect is that business users will also be able to execute their own smart contracts, P2P applications, and other open blockchain apps (DApps) distributed without asking for IT permission. In the same way, SaaS was a Trojan horse that allowed employees to sign for services on their own without disturbing the company's infrastructure. This new form of SaaS can be implemented because a new layer of infrastructure can appear by being supported on a peer-to-peer basis and shared cost. And it is very likely that the cost of this new computing infrastructure will be as cheap as Internet access today, on a per-user basis. This opens the possibilities for further application. Thin cloud represents freedom and flexibility for users and developers. It will allow anyone to create their own business logic for ownership, trade, contract, transaction formats and state transition functions without worrying about setting up an infrastructure. We need to fully embrace the thin cloud as a result of the blockchain's infrastructures, and we need to innovate with the creative applications running on it.

\section{References}

[1] Cloud Computing, Available at https://en.wikipedia.org/wiki/Cloud_computing [January, 2019]

[2] Tiana Laurence, „Introducing Blockchain”, in Blockchain for Dummies, 2017

[3] Lorne Lantz, „What is Bitcoin?" Bitcoin, the Blockchain, and Their Potential to Change Our World

[4] Andrea Monaci, Blockchain on cloud computing: why not? Available at https://www.cloud28plus.com/apac/content/Blockchain-on-cloud-computing--why-not

[5] Ed Featherston, Blockchain as a Service: The New Weapon in the Cloud Wars?, Available: https://dzone.com/articles/blockchain-as-a-service-the-new-weapon-in-the-clou

[6] Vitalik Buterin, William Mougayar, „Blockchain as a Cloud” in The Business Blockchain, 2016

[7] Dan-Cristian Cearnău, Cloud Computing - Emerging Technology for Computational Services, Informatica Economică Journal, vol. 22, no. 4/2018, pp. 61

[8] Jin Ho Park, Jong Hyuk Park, Blockchain Security in Cloud Computing: Use Cases, Challenges, and Solutions, in Symmetry Journal, Advanced in Artificial Intelligence and Cloud Computing, August 2018, Available: https://www.mdpi.com/2073-8994/9/8/164 